satisfactory identification of the arrival of the sky waves has been made.

The equivalent wave-length of an atmospheric may be taken as 17,000-40,000 $\mathrm{m}$. The values of $h$ in the table mean that if $100 \mathrm{~km}$. is the height of the $E$ layer, the conditions of ionization and collision frequency required for the reflection of long waves exist in the upper atmosphere $10-30 \mathrm{~km}$. below the boundary of the $E$ layer as determined by the reflection of short waves. We have sought for evidence of a $D$ or lower ionized layer, but have found none. (If an atmospheric at its source is a single pulse then, as stated above, Fig. 2 could be interpreted as evidence of the existence of low layers, but the balance of evidence, we think, does not support this interpretation.)

An accepted expression for the attenuation of long waves propagated along the ground makes $E . d$ constant, where $E v / m$ is the field strength at a distance $d \mathrm{~km}$. from an electromagnetic radiator of constant power. $E . d$ is found to range in our atmospheric observations from 170 to 350 . This agrees with evidence previously found by C. T. R. Wilson ${ }^{2}$, Munro and Huxley ${ }^{3}$, Boswell and Wark ${ }^{4}$, that a lightning flash does not vary greatly in its initial electric moment and in its radiating power.

In the observations discussed above, the electrical discharge was $70 \mathrm{~km}$. or more from the observing instruments.

T. H. LABY.

F. G. NTOHOLLS.

A. F. B. Nickson.

J. J. MCNEILL.

Natural Philosophy Laboratory,

Melbourne University, Melbourne, N.3.

June 22.

${ }^{1}$ Laby, T. H., Nicholls, F. G., Nickson, A. F. B., and Webster, H. C., NATURE, 139, 837 (1937).

${ }^{2}$ Wilson, C. T. R., Phil. Trans. Roy. Soc., A, 221, 73 (1920).

3 Munro, G., and Huxley, L. G. H., Rept. Aust. R. R. Bd., No. 5 (1932)

- Boswell, R. W., and Wark, W. J., Quart. J. Roy. Met. Soc., 62, 499 (1936).

\section{Time and Probability}

Trme has recently been the subject of much dis. cussion. More than a year ago my colleague, Dr. F. L. Arnot, in connexion with a cosmological theory summarized in NATURE of June 25, directed my attention to the relation between kinematic or atomic time, $t$, and planetary or pendulum time, $\tau$. This may be expressed in the formula

$$
\tau=t_{0} \log \left(t / t_{0}\right)+t_{0},
$$

$t_{0}$ being a constant of integration representing "the present age of the universe at ourselves, reckoned on the $t$-scale" (Milne).

The use of a logarithmic time scale was suggested by de Sitter in 1933, but the further development of such a scale is mainly due to E. A. Milne. Although Milne speaks of $t$ as "probability time" (1937), this aspect of the time scale does not seem to have attracted much attention, and it is the object of this letter to stress the close relationship between time as measured and probability.

Boltzmann's formula

$$
S-S_{0}=k \log \left(W / W_{0}\right),
$$

connecting entropy $S$ and "probability" $W$, is similar in form to that which connects de Sitter's two varieties of time. The entropy formula "corresponds with the fact that entropies are additive and probabilities multiplicative. When a system moves into a more probable state, its entropy is increased and its probability is multiplied by a factor" (Ubbelhode).

The mathematical resemblance between the time formula and the entropy formula is an indication of the fact that kinematic time $t$ may be interpreted as a "probability", and consequently time as measured by an astronomer or physicist is a statistical quantity. Illustrations of this statistical interpretation are not difficult to find. In using a sand glass we may be able to detect on close examination the motion of individual grains of sand, but we note the passage of time by observing the fall of an assemblage of grains. We are dealing with a statistical result and assume that on the average the time taken for the total quantity of sand to pass is the same in successive operations of the hour glass. In the water clock, or clepsydra, the same principle is applicable, but the moving particles are of smaller dimensions. When a 'rigid' body, a pendulum or rotating planet, is used for the measurement of time, we are again concerned, from the point of view of atomic or molecular theory, with a statistical result.

Eddington's picturesque description of entropy as "time's arrow" need not be taken too seriously. In "Philosophy and the Physicists", Stebbing has taken objection to the view that entropy may be regarded as "the signpost of time". The experimenter must be aware of the order of his observations before he can draw conclusions from them. In the majority of his experiments, he is dealing with statistical results. The familiar phrase "the tide of time" is in some measure suggestive of a statistical interpretation of time.

\section{The University, \\ St. Andrews.}

July 21.

\section{Cosmological and Atomic Constants}

IN view of some recent contributions ${ }^{1}$ it may be considered not altogether useless to point out a few more relations (or 'coincidences') between some fundamental magnitudes that occur in astrophysical theory and very large dimensionless numbers that can be constructed from atomic constants and the constant of universal gravitation $G$. If $\gamma_{1}$ and $\gamma_{2}$ represent the large numbers constructed from $\left(e, c, m_{H}, G\right)$ and $\left(\hbar, c, m_{H}, G\right)$ respectively, then

$$
\begin{gathered}
\gamma_{1}=\frac{e^{2}}{m_{H}^{2} G} \sim 1 \cdot 23 \times 10^{36} ; \gamma_{2}=\frac{\hbar c}{m_{B}{ }^{2} G} \sim 1 \cdot 87 \times 10^{33} \\
\text { and } \alpha \equiv \frac{\gamma_{2}}{\gamma_{1}}=\frac{\hbar c}{e^{2}} \sim 137,
\end{gathered}
$$

where $\hbar$ is Planck's constant (multiplied by $1 / 2 \pi$ ), $c$ the velocity of light, $e$ the electron charge, $m_{H}$ the mass of the proton, and $m$ the mass of the electron.

It is known ${ }^{2}$ that the usual theory of the white dwarf stars, when combined with the theory of pressure ionization, predicts a maximum radius for a cold body, that is, a stellar mass composed of matter degenerate in the sense of Fermi-Dirac statistics. Let $R_{\max }$. denote this maximum value of the radius and $M_{0}$ the corresponding mass, then it is interesting to note that the large (astrophysical) dimensionless numbers $M_{0} / M_{B}$ and $R_{\max } /\left(\frac{\hbar}{m c}\right)$ which we can construct from the fundamental magnitudes $M_{0}$ and $R_{\max }$. are very simply related to $\gamma_{1}$ and $\gamma_{\mathrm{z}}$. 